\title{
EPIDEM: A Technology-Enabled COVID-19 Elimination Strategy
}

\author{
Goetz Robert Uwe Grosche1, Sanam Shah², Anna Katrina Javier Bolivar ${ }^{3}$ \\ ${ }^{1}$ I-Optic Computing, Wollongong, Australia \\ ${ }^{2}$ Ecole des Hautes Etudes en Sante Publique (EHESP), Paris, France \\ ${ }^{3}$ Independent Researcher, Quezon City, Philippines \\ Email: rob@i-optic.com, Sanam.shah@hotmail.com, akatrina.jbolivar@gmail.com
}

How to cite this paper: Grosche, G.R.U. Shah, S. and Bolivar, A.K.J. (2020) EPIDEM: A Technology-Enabled COVID-19 Elimination Strategy. E-Health Telecommunication Systems and Networks, 9, 60-69. https://doi.org/10.4236/etsn.2020.94005

Received: November 3, 2020

Accepted: December 12, 2020

Published: December 15, 2020

Copyright $\odot 2020$ by author(s) and Scientific Research Publishing Inc. This work is licensed under the Creative Commons Attribution International License (CC BY 4.0).

http://creativecommons.org/licenses/by/4.0/

\section{(c) (i) Open Access}

\begin{abstract}
Background: The novel Coronavirus disease 2019 (COVID-19) has rapidly evolved into a global emergency with far-reaching consequences. Multiple models predict mounting morbidity and mortality in the absence of appropriate interventions. Screening of sub-clinical cases through technological tools has the potential to eliminate virus containment in the population. Objective: Our aim was to develop an effective application that detected early COVID-19 infections and resulted in four-dimensional (4-D) data visualization systems to develop safe networks whilst respecting user privacy. Methods: We proposed an algorithm for a novel form of contact tracing and screening that can detect infection before manifestation of clinical symptoms and signs. We developed a simulation model that demonstrated impact on the pandemic through percent change in the "trigger point". Results: A "trigger point" based on personal risk assessment generated a visual report to the community network. The following outcomes were rendered in the app: live surveillance of metadata, hotspot mapping, targeted live health messaging to a large population, an infection control passport technology, and personal hotspot avoidance warnings. Our model suggested that higher adoption of such strategies can potentially eliminate viral carriage in the population. Conclusions: Our proposed technology-enabled screening, detection, and elimination strategy presents a novel approach to eliminate the viral containment. This app could be applied to the COVID-19 pandemic as well as other outbreaks and epidemics in the future to control the unprecedented disease spread.
\end{abstract}

\section{Keywords}

Coronavirus Disease 2019, COVID-19, Pandemic, Outbreak, Contact

Tracing, Technology, Application 


\section{Introduction}

As of September 4, 2020, there were 26,016,839 confirmed cases of Coronavirus disease 2019 (COVID-19) with 863,020 deaths [1]. In December 2019, the COVID-19 outbreak declared a worldwide pandemic on March 11, 2020 [2] with social and economic consequences of unimaginable scale.

COVID-19 is mainly a droplet infection transmitted through close contact with infectious people [3]; however, studies have reported potential of transmission via infected surfaces, gastrointestinal and airborne routes as well [4]. Certain segments of the population which include elderly, people with immunodeficiency, and existing cardiovascular, metabolic, and respiratory diseases are at higher risk [5], and the incubation period varies greatly from 2.5 to more than 20 days [6] [7] [8]. Infection was initially categorized into four types: mild, moderate, severe, and critical [9]; however, the risk of transmission of infection from asymptomatic carriers has also been reported [10]. The latter is characterized by absence of clinical and radiological findings but similar infectivity as symptomatic cases [11].

In order to contain an infection, control practices are broadly categorized as follows: controlling the source of infection, cutting off transmission routes, and protecting the at-risk population [12]. Although a vaccine would be the best tool to curb the pandemic by protecting the population atrisk, current clinical trials are in the early investigational phases and will require time until launch and large scale uptake [13]. Another strategy that has potential to slow down viral transmission is timely testing and isolation of infected people. Nevertheless, this can be limited by availability of timely testing and diagnostic accuracy.

A number of existing diseases or conditions put the COVID-19 afflicted population at a greater risk of dying. Nevertheless, there is scarcity of evidence that this heightened predisposition renders them a different trigger point (i.e. a different disease developing timeline) than the rest of the population. Clearly, the at-risk population needs special and more urgent attention to meet higher costs and infrastructure needs than the rest of the population. In this regard, prediction modeling offers a promising decision-making field for detection, diagnosis, and prognosis of infection [14]. Prediction models have the advantage of combining several variables to predict risk of infection and assist in triaging people. Several models based on rule based scoring systems to advanced machine learning models have been proposed recently to inform public health and protect lives [15]. In addition, a summary of the World Health Organization (WHO) surmise that the research to formulate historic data modeling is sound and carries high credibility [16].

Therefore, we propose a novel "EPIDEM" rapid prediction containment strategy to proactively detect potentially infectious people in the pre-clinical stage in order to implement protective and preventive steps that could quickly eliminate infection at large. Moreover, generation of a contact infection-risk passport would aid in limiting spread of infection. We anticipate that the early detection 
of potential cases based on personal criteria would encourage testing and isolation in a timely and safe manner.

\section{Methodology}

The proposal of this approach was based on the findings that asymptomatic infections were a barrier to containment of the infection, and implementation of isolation was the best option for asymptomatic carriers [17] and containment of infection [10]. The model was based on the ability to predict infection trends in select towns based on the person's current health condition to risk-stratify as early as possible.

\subsection{Hypothesis}

The authors hypothesize that the EPIDEM can be help build social networks of uninfected contacts and infection-free hotspots in a population to aid COVID-19 elimination.

\subsection{The Simulation Model}

We utilized the following key clinical pointers of a patient's infectiousness: temperature, dry cough, expectorant, sore throat, shortness of breath, nausea, vomiting, diarrhea, fatigue, arthralgia, and having pale, clammy skin [18]. In order to set the weighting for the EPIDEM trigger point, the order of symptom development was taken into account as follows: fever, cough, nausea and/or vomiting followed by diarrhea [19]. Furthermore, comorbidity data was taken into account based on a previous study which has shown high risk for COVID-19 mortality associated with diabetes [20].

Users would assess their risk by accessing the app. Data provided by the users would quantify their level of risk and an appropriate notification would be displayed on the screen. EPIDEM would enable a four-dimensional (4-D) monitoring and outbreak visualization with early identification of the infection. EPIDEM-V1 would allow visualization of groups and hotspots, and generation of a new passport for lay people and public health professionals whereas EPIDEM-V2 would provide the COVID-19 elimination strategy technology. Following identification of an infected person, the EPIDEM-V1 map would display a geographic increase in symptoms and the social link of EPIDEM-V2 would aid in rapid identification of linked contacts.

The outbreak detection monitor and trigger would show the rate of change from asymptomatic to various stages of infection. Furthermore, the rate of change over time of early symptoms would be the trigger point to isolate patients quickly. It would appear at the earliest point in the infectious cycle.

The COVID-19 infection status passport, an epidemiological artificial intelligence (AI) assessment tool, would give weighted advice to individuals and metadata/exact data to public health professionals. The advice would be displayed in a graph and a color-coded traffic light system. 


\section{Results}

The COVID-19 Eradication Technology App (http://epideminfo.us/) [21] enables visualization of 4-D epidemiological outbreaks and instant identification of outbreak location, scale, speed, and stage. The geographic presentation of outbreaks allows users to know which places to avoid and also allows people from the health authority to know which areas to prioritize. The metadata gives live data, which can be very useful as basis for decision making during outbreak response.

\section{Principal Findings}

1) We visualized the onset of symptoms with the daily epidemiological EPIDEM (V1 and V2) map of an at-risk population.

2) We analyzed the percent change in individual health weighting caused by COVID-19 using screening questions. The trigger to detect the onset of symptoms was the increase in a person's health weighting for two or more consecutive days or a single day of unusual upward change.

3) We developed the algorithm for preliminary detection of COVID-19 from data and the structured query language (SQL) detected people with worsening symptoms. This group of people was color graded and added to a Google heatmap.

4) The app rendered the following outcomes: live surveillance of metadata, hotspot mapping, targeted live health messaging to a large population, an infection control passport technology, and personal hotspot avoidance warnings.

\section{Discussion}

With the start of the pandemic, many novel applications were developed in order to control the rapid spread of COVID-19 infection. Apps served diverse public health needs such as epidemiological surveillance, rapid case identification, interruption of community transmission, public communication and provision of clinical care [22]. The adoption of individualized risk prediction models to inform decision making and clinical care is increasing with time. Furthermore, these applications serve an additional advantage of providing many novel findings such as the association of the risk of infection with socio-economic status, ethnicity, and medical history such as prior flu and pneumococcal vaccination [23].

Data visualization tools for decision making that synthesize real time public health data have also gained popularity in keeping the public informed [24] [25]. Some of the apps are based on time series data from region-level to case-level [25]. In case of time and region dependent data, model recalibration is also of prime importance [23]. Nevertheless, there seems to be a lack of data on effectiveness of contact-tracing apps in literature therefore the quality and consistency of data remains of concern.

A review of literature showed that apps in use in the UK [26] and Singapore [27] served as good surveillance tools that monitored infections; however, this is 
of limited utility in case of asymptomatic infections. Hence, digital contact tracing tools have gained importance in larger populations. Such technologies have been implemented in China [28], South Korea [29], and Norway [30]. One of the limitations of digital contact tracing is the need for a large population to use the app. For instance, there was merely $30 \%$ reported uptake of a tracer app in Singapore [31]. Key practical issues remained such as differentiating clinical COVID-19 infections from other respiratory illnesses, understanding of contacts that were deemed to be close enough for transmission, and determining adequate exposure time that was considered long enough to trigger an alert.

In our present work, through integration of live data and user privacy-protected metadata, we provided visualization of the evolution of the epidemic and effectiveness of a four-pillared eradication technology, namely COVID-19 protection, avoidance, detection, and surveillance. Our findings indicated that creation of COVID-19 free hotspots had the potential to allow faster reopening of socioeconomic activities and workplaces.

\subsection{EPIDEM as a Tool for Early Case Identification}

Epidemiology is a great tool for modeling historical data and the core engine of EPIDEM makes use of it for risk predictions. Our proposed app-based strategy would detect infections at the earliest point in time. Since many infections occur in the preclinical stage before the onset of any symptoms or signs, isolating people after the development of symptoms would lose the window of opportunity. Hence, potential cases should be detected before symptom manifestation to reduce spread of infection. In addition, reducing proximity to potential cases is important. The risk of infection is greatest within 1.5 - 2 meters for at least 10 15 minutes [32]. Our mathematical model of tracing showed fast tracking which has the potential to eliminate viral carriage in the community and resolving large scale lockdown measures.

\subsection{EPIDEM Uses the Same Infection Symptom Trigger Point for the Entire Population}

So far, diverse symptom and risk screening applications have been adopted worldwide. Screening effectiveness varies considerably with some sources suggesting less than 50\% detection amidst the growing epidemic [33] [34]. Screening can also be limited by the ineffectiveness to detect individuals early in the incubation period and those early on after the onset of symptoms. This is further complicated by individual variation in the incubation period. To improve the effectiveness of screening, we utilized the following strategies: the natural history of infection with increasing likelihood to detect symptoms with the time since first exposure, and developing the same infection trigger point for the entire population. EPIDEM supplied high quality live epidemiological data in infection zones and accounted for the percentage of subclinical cases that were likely to be missed. We accounted for the user's age to address age discrepancies in mortality 
which is highest in the elderly [20], and age-related variations in sub-clinical cases.

\subsection{EPIDEM Supplies Information to Resource the Health Response in Accordance with Medically Predisposed Population Counts in an Infection Zone}

Real-time disease surveillance at population level through aggregate and individual-level data would deliver scientific resources to educate the public about COVID-19 and information on avoidance of infection via social distancing and other measures. Moreover, aggregated data would deliver detailed visualization maps that would highlight areas of potential high number of cases, connect individuals with low risk, and create healthy connected communities.

\subsection{EPIDEM Can Supply Relevant Information Updates to at Risk Populations (as Advised by Professionals i.e. Higher Morbidity in Population with Preexisting Conditions)}

Evidence suggests that certain conditions such as cardiovascular diseases, diabetes and obesity, predispose individuals to higher risk of morbidity and poor outcomes [35]. Risk prediction through integration of personal assessment would deliver tailored information to individuals at high risk due to COVID-19. In order to address the question of the at-risk population, we clarified "existing conditions" and did a statistical count of patients in an at-risk area. EPIDEM can supply this data live and updated on a daily basis which would be beneficial for health resource allocation as well.

\subsection{EPIDEM Harness the Power of Lay Epidemic Crowd Vigilance (as Defined by Professionals)}

Public support and active participation of users can be substantially harnessed for data processing with significant results. The data on proximity of two individuals would be enough to derive risk assessments. EPIDEM makes use of the vigilance of the entire population to spot outbreaks, and to assist in isolating and referring very early symptomatic patients to health care workers.

\subsection{EPIDEM Uses Social Media Crowd Vigilance to Assist in Infection Spotting and Quarantining Compliance (as Defined by Professionals)}

Modern technologies have immensely aided people during this pandemic and use of social media has the potential to greatly encourage community-wide containment and elimination of the virus. This would be achieved via data surveillance, targeted decision making about quarantine of potentially exposed contacts, and encouragement of health checks.

\subsection{EPIDEM Applies Social Media Pressures for Policing and Quarantining Compliance (as Defined by Professionals)}

Social media epidemiology is a powerful policing tool because friends, family, 
neighbors, and business contacts can watch each other to keep themselves safe in a social media circle of trust and vigilance. This is a lay-grassroots epidemiological vigilance which assists health professionals in containing outbreaks, tracking patients, and leading to EPIDEM being a COVID-19 eradication and strategy tool. We utilized an effective approach to enforce quarantine rules by notification to exposed persons and warnings before entering "high-risk zones". If the person would be in close proximity to a potentially infected user, it would lead to generation of a notification to go into quarantine at home. Furthermore, the user can take further steps to contact local health departments to get tested for COVID-19 as soon as possible to determine the further course of action. Hence, we anticipate a more effective contact tracing system complying with existing guidelines.

\subsection{EPIDEM Adds a Live Four-Dimensional Visualization Surveillance Tool to an Epidemiologist's Arsenal of Tools}

Our epidemiological model where one is considered to be potentially infectious or susceptible to infection would systematically produce updated data across time to visualize spread of infection. Individuals may move to a new point at each unit in time from the prior contact point. As EPIDEM uses historic modeling reduced to a weighting algorithm, this algorithm would be adjusted when new historic data changes the weighting of evidence with the aim to supply the best extrapolation to help minimize the current outbreak.

\section{Conclusions}

The newly developed technology, EPIDEM, has a lot of features that can enable a country to contain COVID-19. It can aid for early case identification; it can aid in quarantine compliance; it can provide relevant and timely data to health professionals; and it can serve as an epidemiological surveillance tool.

The EPIDEM application has the ability to fight the rapid transmission of COVID-19 and similar epidemics and pandemics in the future. With increasing digitalization in the COVID-19 era, we anticipate similar health technologies to be on the forefront to fight healthcare challenges in the future.

\section{Acknowledgements}

The authors would like to acknowledge the team of people who shared their expertise for the development of EPIDEM. We would like to thank Veit Grosche, Layla McNeil, Haris Fawad, Muhammad Waseemwalich, Mark Kwagbedzie, Tapan Kumar, and Khriz Jayce Perey. We would also like to thank the programmers who worked hard to help develop EPIDEM.

\section{Conflicts of Interest}

The authors declare no conflicts of interest regarding the publication of this paper. 


\section{References}

[1] World Health Organization Coronavirus Disease (COVID-19) Dashboard. https://covid19.who.int

[2] World Health Organization (2020) Coronavirus Disease (COVID-19) Outbreak. https://www.euro.who.int/en/health-topics/health-emergencies/coronavirus-covid-19

[3] Q\&A: How Is COVID-10 Transmitted? https://www.who.int/emergencies/diseases/novel-coronavirus-2019/question-and-a nswers-hub/q-a-how-is-covid-19-transmitted?gclid=EAIaIQobChMIjqWp653F6wlI VV7LVCh1XVQ7ZEAAYASAAEgKbm_D_BwE

[4] Xiao, F., Tang, M., Zheng, X., Liu, Y., Li, X. and Shan, H. (2020) Evidence for Gastrointestinal Infection of SARS-CoV-2. Gastroenterology, 158, 1831-1833.e3. https://doi.org/10.1053/j.gastro.2020.02.055

[5] Team CC-19 R, Centers for Disease Control and Prevention (CDC) COVID-19 Response Team, Bialek, S., Boundy, E., Bowen, V., Chow, N., Cohn, A., Dowling, N., Ellington, S., Gierke, R., et al. (2020) Severe Outcomes among Patients with Coronavirus Disease 2019 (COVID-19)-United States, 12 February-March 16, 2020. Morbidity and Mortality Weekly Report, 69, 343-346. https://doi.org/10.15585/mmwr.mm6912e2

[6] Liu, W., Wang, F., Li, G., Wei, Y., Li, X., He, L., Yue, H., Zhang, F., Hu, Q., Chu, J., et al. (2020) Analysis of 2019 Novel Coronavirus Infection and Clinical Characteristics of Outpatients: An Epidemiological Study from the Fever Clinic in Wuhan, China. SSRN Electronic Journal.

[7] Yang, W., Cao, Q., Qin, L., Wang, X., Cheng, Z., Pan, A., Dai, J., Sun, Q., Zhao, F., Qu, J., et al. (2020) Clinical Characteristics and Imaging Manifestations of the 2019 Novel Coronavirus Disease (COVID-19): A Multi-Center Study in Wenzhou City, Zhejiang, China. Journal of Infection, 80, 388-393. https://doi.org/10.1016/j.jinf.2020.02.016

[8] Guan, W.-J., Ni, Z.-Y., Hu, Y., Liang, W.-H., Ou, C.-Q., He, J.-X., Liu, L., Shan, H., Lei, C.-L., Hui, D.S.C., et al. (2020) Clinical Characteristics of Coronavirus Disease 2019 in China. The New England Journal of Medicine, 382, 1708-1720. https://doi.org/10.1056/NEJMoa2002032

[9] Yuki, K., Fujiogi, M. and Koutsogiannaki, S. (2020) COVID-19 Pathophysiology: A Review. Clinical Immunology, 215, Article ID: 108427. https://doi.org/10.1016/j.clim.2020.108427

[10] Gao, Z., Xu, Y., Sun, C., Wang, X., Guo, Y., Qiu, S. and Ma, K. (2020) A Systematic Review of Asymptomatic Infections with COVID-19. Journal of Microbiology, Immunology and Infection. https://doi.org/10.1016/j.jmii.2020.05.001

[11] Lao, X., Luo, L., Lei, Z., Fang, T., Chen, Y., Liu, Y., Ding, K., Zhang, D., Wang, R., Zhao, Z., et al. (2019) Epidemiological Characteristics and the Effectiveness of Countermeasures to Control Coronavirus Disease 2019 in Ningbo City, China.

[12] van Seventer, J.M. and Hochberg, N.S. (2017) Principles of Infectious Diseases: Transmission, Diagnosis, Prevention, and Control. In: International Encyclopedia of Public Health, Elsevier, Boston, 22-39.

https://doi.org/10.1016/B978-0-12-803678-5.00516-6

[13] Treatments and a Vaccine for COVID-19: The Need for Coordinating Policies on R\&D, Manufacturing and Access.

https://www.oecd.org/coronavirus/policy-responses/treatments-and-a-vaccine-for-covi d-19-the-need-for-coordinating-policies-on-r-d-manufacturing-and-access-6e7669a 9

[14] Wynants, L., Van Calster, B., Collins, G.S., Riley, R.D., Heinze, G., Schuit, E., Bon- 
ten, M.M.J., Damen, J.A.A., Debray, T.P.A., De Vos, M., et al. (2020) Prediction Models for Diagnosis and Prognosis of Covid-19 Infection: Systematic Review and Critical Appraisal. BMJ, 369, m1328. https://doi.org/10.1101/2020.03.24.20041020

[15] Sharing Research Data and Findings Relevant to the Novel Coronavirus (COVID-19) Outbreak 2020.

https://wellcome.ac.uk/press-release/sharing-research-data-and-findings-relevant-n ovel-coronavirus-covid-19-outbreak

[16] COVID-19 and Contact Tracing. https://extranet.who.int/goarn/sites/default/files/What\%20are\%20best\%20practices \%20for\%20contact $\% 20$ tracing\%20for\%20COVID19\%20SARS\%20MERS\%20and $\% 2$ 0influenza.pdf

[17] Yu, X. and Yang, R. (2020) COVID-19 Transmission through Asymptomatic Carriers Is a Challenge to Containment. Influenza and Other Respiratory Viruses, 14, 474-475. https://doi.org/10.1111/irv.12743

[18] de O Lima, C.M.A. and de Oliveira Lima, C.M.A. (2020) Information about the New Coronavirus Disease (COVID-19). Radiologia Brasileira, 53, 5-6. https://doi.org/10.1590/0100-3984.2020.53.2e1

[19] Larsen, J.R., Martin, M.R., Martin, J.D., Kuhn, P. and Hicks, J.B. (2020) Modeling the Onset of Symptoms of COVID-19. Frontiers in Public Health.

https://doi.org/10.3389/fpubh.2020.00473

[20] Caramelo, F., Ferreira, N. and Oliveiros, B. (2020) Estimation of Risk Factors for COVID-19 Mortality_Preliminary Results. Cold Spring Harbor Laboratory Press, Cold Spring Harbor. https://doi.org/10.1101/2020.02.24.20027268

[21] EPIDEM. http://epideminfo.us

[22] Budd, J., Miller, B.S., Manning, E.M., Lampos, V., Zhuang, M., Edelstein, M., Rees, G., Emery, V.C., Stevens, M.M., Keegan, N., et al. (2020) Digital Technologies in the Public-Health Response to COVID-19. Nature Medicine, 26, 1183-1192. https://doi.org/10.1038/s41591-020-1011-4

[23] Jehi, L., Ji, X., Milinovich, A., Erzurum, S., Rubin, B.P., Gordon, S., Young, J.B. and Kattan, M.W. (2020) Individualizing Risk Prediction for Positive Coronavirus Disease 2019 Testing: Results from 11,672 Patients. Chest, 158, 1364-1375. https://doi.org/10.1016/j.chest.2020.05.580

[24] Ministry of Health Singapore (2020) Updates on Covid-19 (Coronavirus Disease 2019) Local Situation. https://www.moh.gov.sg/covid-19

[25] Latest Situation of Novel Coronavirus Infection in Hong Kong. Centre for Health Protection, Department of Health. The Government of the Hong Kong Special Administrative Region. https://chp-dashboard.geodata.gov.hk/covid-19/en.html

[26] NHS 111 Online (2020) United Kingdom National Health Services. https://111.nhs.uk/covid-19

[27] (2020) Singapore COVID-19 Symptom Checker. https://sgcovidcheck.gov.sg

[28] Bonsall, D. and Fraser, C. (2020) Sustainable Containment of COVID-19 Using Smartphones in China: Scientific and Ethical Underpinnings for Implementation of Similar Approaches in Other Settings.

https://int.nyt.com/data/documenthelper/6825-coronavirus-app-proposal-UK/7665 0ed3f249bf888f1e/optimized/full.pdf

[29] Zastrow, M. (2020) South Korea Is Reporting Intimate Details of COVID-19 Cases: Has It Helped? Nature. https://doi.org/10.1038/d41586-020-00740-y

[30] Halt to COVID-19 Contact Tracing App a Major Win for Privacy. Amnesty Inter- 
national.

https://www.amnesty.org/en/latest/news/2020/06/norway-covid19-contact-tracingapp-privacy-win

[31] (2020) Singapore Government Agency Website. TraceTogether. https://www.tracetogether.gov.sg

[32] Abeler, J., Bäcker, M., Buermeyer, U. and Zillessen, H. (2020) COVID-19 Contact Tracing and Data Protection Can Go Together. JMIR mHealth and uHealth, 8, e19359. https://doi.org/10.2196/19359

[33] Niehus, R., De Salazar, P.M., Taylor, A. and Lipsitch, M. (2020) Quantifying Bias of COVID-19 Prevalence and Severity Estimates in Wuhan, China That Depend on Reported Cases in International Travelers.

[34] Bhatia, S., Imai, N., Cuomo-Dannenburg, G., Baguelin, M., Boonyasiri, A., Cori, A., Cucunubá, Z., Dorigatti, I., FitzJohn, R., Fu, H., Gaythorpe, K., Ghani, A., Hamlet, A., Hinsley, W., Laydon, D., Nedjati-Gilani, G., Okell, L., Riley, S., Thompson, H., Elsland, S., Volz, E., Wang, H., Wang, Y., Whittaker, C., Xi, X., Donnelly, C. and Ferguson, N. (2020) Report 6: Relative Sensitivity of International Surveillance. Imperial College.

https://www.imperial.ac.uk/mrc-global-infectious-disease-analysis/covid-19/report6-international-surveillance/

[35] Centers for Disease Control and Prevention (2020) Coronavirus Disease 2019 (COVID-19).

https://www.cdc.gov/coronavirus/2019-ncov/need-extra-precautions/people-withmedical-conditions.html 